\title{
Ship Mooring Optimization Based on Genetic Algorithm and BP Neural Network
}

\author{
Xiaoying $\mathrm{Xu}^{1, \text { a }}$, Kuan Wang ${ }^{2, b}$ \\ 1, Wenhua College, Wuhan, China \\ ${ }^{2}$ China Classification Society Wuhan Branch, Wuhan, China \\ axuxiaoying52616@163.com , bk_wang@ccs.org.cn
}

Keywords: Mooring optimization; Moses; Time domain; BP neural network; Genetic algorithm Abstract. According to a pipe laying vessel in the South China Sea, we can get different mooring layouts with anchor distance and angle as factors of the orthogonal test. Then the stress and motion displacement of anchor based on different layouts are calculated by using Moses software in different wave direction. The above results are taken as samples, and the BP neural network is trained to replace the Moses time domain calculation. Take the anchor distance and angle as the optimization variable and the weighted translational displacement of different wave direction as optimization objective. Finally, by using genetic algorithm the translational displacement of the vessel at each wave direction significantly reduced. The optimization effect is obvious, and it provides a reference for the mooring arrangement of offshore floating structures.

\section{Introduction}

As we all know in order to maintain a fixed position on the sea, the ship or floating platform must use a mooring system. When the mooring displacement is too large, the normal operation is affected; when the mooring line layout is not reasonable, it is easy to cause fracture. Therefore reducing the translational displacement and reasonable layout of mooring line are particularly important. After the proposed model of multi-component mooring line based on multi-objective optimization, Yu Long, Tan Jiahua [1] completed the design of mooring system according to the genetic algorithm combined with Atlas Design; Ma Xiaojian, Sun Zhaochen [2] studied the tension and translation displacement of mooring line under the action of wind and current; Based on the assume that each mooring line is exactly the same, Goo, J., Yoshida, K. [3] optimized of the cable ship length of the line and relative angle between line and the vessel by adopting genetic algorithm.

On the basis of some meaningful conclusions obtained by the above scholars, we studied the pipe laying vessel working in the South China Sea from the practical point of view. Considering the influence of environmental, we used the anchor position and angles as the optimization variable, and optimized the layout of the pipe. The main contents of this paper are as follows: (1) According to the drawings of the pipe laying ship, we conducted frequency domain analysis through using the model by Moses. After the hydrodynamic coefficient is obtained, the results are transformed into the time domain. Considering the interaction between the hull and anchor, we calculated the conventional the movement and force of cable by using the time domain method based on Newmark integral under the condition of wind and current. (2) According to the principle of orthogonal test, we can get 32 mooring layouts by taking 4 levels on the distance and angle of the anchor chain. The floating motion and the force under each different layout are obtained through the preparation of the batch file, and all of the results simulated as training sample of BP neural network. (3) By using genetic algorithm the translational displacement of the vessel at each wave direction significantly reduced.

\section{Principle of BP Neural Network and Genetic Algorithm}

BP Neural Network. BP neural network is famous for its strong approximation and promotional ability. It can be used to deal with complex nonlinear and uncertain objects with the coordination of a variety of relationships of multiple samples. Input layer, hidden layer and output layer are concluded. 
The layers are connected by weights, and each layer contains some neurons which each attaches a threshold. A map is formed between the input and output of the network, as shown in Fig.1.

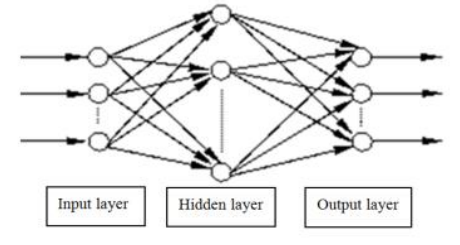

Fig.1 BP Neural Network

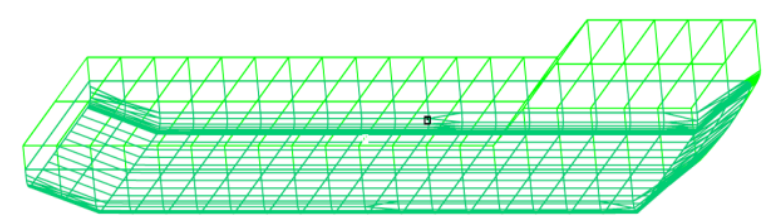

Fig.2 Model based on Moses

Generally, We chose Tansig function in the hidden layer and Purelin function in the output layer as the transfer function to build BP neural network. Eq. 1 shows the new model. All of the meaning of symbol in Eq.1 6 can be seen from Tab.1.

$$
O_{i}=\sum_{j=1}^{J} W_{i j} a\left(\sum_{k=1}^{K} W_{j k} \tanh \left(\sum_{n=1}^{N} W_{k n} \xi_{n}+b_{1 k}\right)+b_{2 j}\right)+b_{3 i}
$$

The error is defined as the difference between the desired and actual output of the network, and it transmits step by step from output to forward. In the process, the network weight is adjusted by the error, and ultimately made its permissible range of error. The error function is shown in Eq.2.

$$
E_{P}=\frac{1}{2} \sum_{j=1}^{m}\left(t_{j}^{P}-y_{j}^{P}\right)^{2}
$$

There are many training algorithms in the network toolbox of MATLAB. By comparison, Traingdm was chosen because of its smaller error and shorter training time. In addition, this method accelerated convergence by correcting some parameters and could search the global optimum quickly.

Genetic Algorithm. The object is a group of binary strings. Firstly, we selected the individual from the initial population which met the fitness function. Then, the next generation of population was produced through the hybrid and mutation. Finally, the calculation ended until the expected conditions met. The process is shown in Fig.3.

Genetic algorithm toolbox in MATLAB includes various sub-functions as follows.

For the population initialization function, We chose the crtbp function because of its more convenient in the realization of coding, hybrid and mutation. It is shown in Eq.3.

Chrom $=\operatorname{crtrp}$ （numind, lenind )

The fitness function is mainly used to evaluate the merits and demerits of individuals, and the higher the fitness value is, the higher the probability of individual inheritance next generation. Because the ranking function, which we chose, is based on the ranking of the fitness allocation and it supports for non-linear assessment. It is shown in Eq.4

FitV=ranking $(\mathrm{Obj} \mathrm{V})$

In the above formula, the larger the $\mathrm{ObjV}$ value, the smaller the fitness value is assigned.

Selection function is used to carry out the survival of the fittest, we chose the advanced entry function (select) to facilitate multi-population interface, shown in Eq.5

SelChr $=\operatorname{select}($ SEL_F, Chrom, FitnV, GGAP)

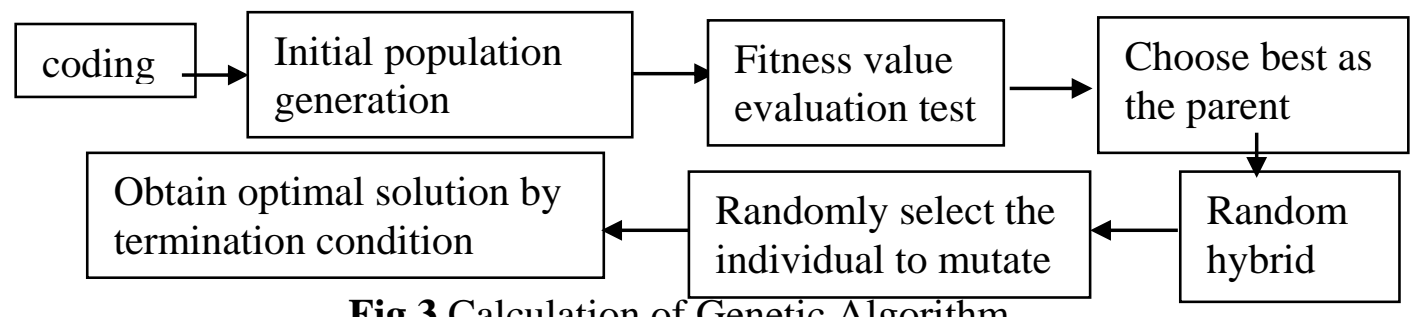

Fig.3 Calculation of Genetic Algorithm

New individuals can not be produced only through the selection, so hybrid-operation is needed. We chose the advanced entry function (recombin), because it provides multiple subgroup support for all hybrid operations, shown in Eq.6.

NewChr = recombine $($ REC_F, Chrom, RecOpt $)$ 
Tab.1 Meaning of Symbol

\begin{tabular}{|l|l|c|l|l|l|l|l|}
\hline Symbol & Meaning & Symbol & Meaning & Symbol & Meaning & Symbol & Meaning \\
\hline $\begin{array}{l}\xi_{\mathrm{n}} \\
\boldsymbol{O}_{i}\end{array}$ & $\begin{array}{l}\text { Input } \\
\text { output }\end{array}$ & $\begin{array}{l}b_{1 k}, b_{2 j}, b_{3 i} \\
t_{j}{ }^{P}\end{array}$ & $\begin{array}{l}\text { threshold } \\
\text { Desired } \\
\text { output }\end{array}$ & REC_F & $\begin{array}{l}\text { A string containing } \\
\text { low-level } \\
\text { reorganization } \\
\text { function }\end{array}$ & $\begin{array}{l}\text { RecOpt } \\
\text { hybrid } \\
\text { probability } \\
\text { taking 0.7) }\end{array}$ \\
\hline $\mathrm{P}$ & $\begin{array}{l}\text { Num of } \\
\text { samples }\end{array}$ & $y_{j}{ }^{P}$ & $\begin{array}{l}\text { Actual } \\
\text { output }\end{array}$ & $\begin{array}{l}\text { numindle } \\
\text { nind }\end{array}$ & $\begin{array}{l}\text { Individual number } \\
\text { \& length }\end{array}$ & GGAP & $\begin{array}{l}\text { Generation } \\
\text { gap }(0.9)\end{array}$ \\
\hline$W_{k n}, W_{j k}, W_{i}$ & weight & FitnV & $\begin{array}{l}\text { fitness } \\
\text { value }\end{array}$ & SEL_F & Name of function & Chrom & $\begin{array}{l}\text { Population } \\
\text { chosen }\end{array}$ \\
\hline
\end{tabular}

As the choice and cross-operation may cause the loss if some information, mutation operator function is needed to ensure the effectiveness of the algorithm. We chose the mut function as mutation operator.

\section{Example Analysis}

Background. In this paper, we calculated time domain motion and force value of the anchor of the pipe laying vessel, which operates for the South China Sea with galvanized steel wire rope. Main parameter and the environmental conditions are shown in Table 2 below. The angle of cable indicates the angle between the cable and $\mathrm{X}$ axis in the hull coordinate system and points to the $\mathrm{Y}$ axis is positive.

Initial analysis through Moses software simulated the stiffness changes at different locations with the time domain module, which can be carried out by the roll damping correction. According to the hull profile, the shape of each station of the hull is established, which formed the surface. As the ship line is more regular, so the maximum side length of grid is set to 12 meters. The program automatically divided into 678 faces after the formation. Hydrodynamic model was shown in Fig.3.

Tab.2 Main Parameter of vessel and environment

\begin{tabular}{|c|c|c|c|}
\hline name & value & name & value \\
\hline $\mathrm{L}_{\mathrm{pp}}$ & $168 \mathrm{~m}$ & Water depth & $190 \mathrm{~m}$ \\
\hline $\mathrm{B}$ & $46 \mathrm{~m}$ & Wave height & $2.5 \mathrm{~m}$ \\
\hline $\mathrm{D}$ & $13.5 \mathrm{~m}$ & speed & 1.08 \\
\hline $\mathrm{D}_{\mathrm{w}}$ & $21400 \mathrm{t}$ & Wind speed & 16 \\
\hline Gravity center & $77.12,0.42,10.4$ & Wave cycle & $12 \mathrm{~s}$ \\
\hline $\mathrm{L}_{\text {cable }}$ & $2500 \mathrm{~m}$ & diameter & $76 \mathrm{~mm}$ \\
\hline Break force & $3800 \mathrm{KN}$ & number & 8 \\
\hline Winch pull & $1100 \mathrm{KN}$ & & \\
\hline
\end{tabular}

Tab.3 Calculation under Different Pre-tension

\begin{tabular}{|c|c|c|c|c|}
\hline \multicolumn{2}{|c|}{ Pre-tension } & 30 & 50 & 70 \\
\hline \multirow{3}{*}{ Surge(m) } & Mean square & 6.524 & 3.326 & 2.053 \\
\cline { 2 - 5 } & Righteousness & -7.367 & -4.168 & -5.186 \\
\cline { 2 - 5 } & $\max$ & -8.719 & -2.843 & -3.815 \\
\hline \multirow{3}{*}{ Sway(m) } & Mean square & 0.071 & 0.049 & 0.021 \\
\cline { 2 - 5 } & Righteousness & -0.098 & -0.075 & -0.037 \\
\cline { 2 - 5 } & $\max$ & -0.114 & -0.089 & -0.061 \\
\hline
\end{tabular}

After the impulse response function is obtained through Fourier transforming from the frequency response function, the time-domain motion response is got through convolution integral based on the pseudo-random wave formed by JONSWAP spectrum. The time length is set to 4000s (The result of the first $500 \mathrm{~s}$ is neglected), and the step is $0.2 \mathrm{~s}$. The time domain results of the hull under different pre-tension can be obtained. As shown in Tab.3. It can be seen from the above, all values meet the standard of strength. And Moses calculation results are credible. 
Build the Network. We used the anchor angle and the distance as the factors of orthogonal test. Because the anchor chain is arranged symmetrically along the length of the ship, the anchor chain on one side of ship can be selected. The number of factors is reduced from 16 to 8 , which greatly reduces the workload. We took four levels for each factor. According to engineering experience, the distance was selected $850 \mathrm{~m}, 1000 \mathrm{~m}, 1500 \mathrm{~m}, 2000 \mathrm{~m}$. The azimuth angle of anchor chain S2 is set to $95^{\circ}, 105^{0}$, $120^{\circ}, 135^{\circ}$ and S4 is set to $5^{0}, 15^{\circ}, 30^{\circ}, 45^{\circ}$, so that the anchor chain can be set in counterclockwise without staggering. The angles of S1 and S3 are determined according to S2 and S4, and the four levels of the angles are $10^{\circ}, 20^{\circ}, 30^{\circ}$, and $40^{\circ}$. The orthogonal table L32 $\left(4^{9}\right)$ is shown in Tab.4.

In view of the great number of experimental groups, which each group took about 1 hour, we got the translational displacement and the force under the angle of $0^{\circ} 、 45^{\circ} 、 90^{\circ} 、 135^{\circ} 、 180^{\circ}$ through the preparation of batch documents. Due to space constraints, the result of $0^{0}$ was in Tab.5.

Tab.4 Time Domain Calculation under $0^{0}$ waves

\begin{tabular}{|c|c|c|c|c|c|c|c|c|c|c|c|c|c|c|c|c|c|c|}
\hline \multirow{2}{*}{$\begin{array}{l}\mathrm{nu} \\
\mathrm{m}\end{array}$} & \multicolumn{4}{|c|}{$\begin{array}{l}\text { Anchor displacement } \\
\text { (m) }\end{array}$} & \multicolumn{4}{|c|}{$\begin{array}{c}\text { Angle of chain } \\
\left({ }^{\circ}\right)\end{array}$} & \multicolumn{2}{|c|}{$\begin{array}{l}\text { translationaldispla } \\
\text { cement }(\mathrm{m})\end{array}$} & \multicolumn{8}{|c|}{$\begin{array}{l}\text { force } \\
\text { (ton) }\end{array}$} \\
\hline & MS1 & MS2 & MS3 & MS4 & S1 & S2 & S3 & S4 & Sway & Surge & P1 & P2 & P3 & P4 & S1 & S2 & S3 & S4 \\
\hline 1 & 850 & 850 & 850 & 850 & 105 & 95 & 15 & 5 & 0.04 & -4.18 & 35.2 & 32.2 & 20.2 & 19.9 & 35.2 & 32.2 & 20.2 & 19.9 \\
\hline 2 & 850 & 1000 & 1000 & 1000 & 125 & 105 & 35 & 15 & 0.02 & -5.29 & 32.6 & 31.3 & 29.0 & 28.9 & 32.6 & 31.3 & 29.0 & 28.9 \\
\hline 3 & 850 & 1500 & 1500 & 1500 & 150 & 120 & 60 & 30 & 0.00 & -7.91 & 24.1 & 26.5 & 36.3 & 42.2 & 24.1 & 26.5 & 36.2 & 42.2 \\
\hline 4 & 850 & 2000 & 2000 & 2000 & 175 & 135 & 85 & 45 & 0.00 & -14.93 & 15.9 & 18.9 & 33.4 & 60.2 & 15.9 & 18.9 & 33.3 & 60.2 \\
\hline 5 & 1000 & 850 & 850 & 1000 & 160 & 120 & 45 & 15 & 0.01 & -5.83 & 26.9 & 28.3 & 3541 & 37.8 & 26.9 & 28.3 & 35.3 & 37.8 \\
\hline 6 & 1000 & 1000 & 1000 & 850 & 165 & 135 & 45 & 5 & 0.01 & -5.87 & 25.8 & 26.9 & 3.47 & 40.0 & 25.8 & 26.9 & 36.4 & 40.01 \\
\hline 7 & 1000 & 1500 & 1500 & 2000 & 115 & 95 & 55 & 45 & 0.01 & -7.62 & 31.1 & 30.7 & 0.35 & 30.3 & 31.1 & 30.7 & 30.3 & 30.3 \\
\hline 8 & 1000 & 2000 & 2000 & 1500 & 115 & 105 & 50 & 30 & 0.01 & -6.57 & 31.5 & 31.0 & 29.9 & 29.8 & 31.5 & 31.0 & 29.9 & 29.8 \\
\hline 9 & 1500 & 850 & 1000 & 1500 & 125 & 95 & 65 & 45 & 0.00 & -9.32 & 27.5 & 30.1 & 33.8 & 36.2 & 27.5 & 30.1 & 33.8 & 36.2 \\
\hline 10 & 1500 & 1000 & 850 & 2000 & 145 & 105 & 40 & 30 & 0.01 & -5.79 & 29.3 & 29.9 & 33.3 & 33.6 & 29.3 & 29.9 & 33.3 & 33.6 \\
\hline 11 & 1500 & 1500 & 2000 & 850 & 130 & 120 & 55 & 15 & 0.01 & -6.63 & 28.2 & 28.6 & 33.6 & 37.1 & 28.2 & 28.6 & 33.6 & 37.1 \\
\hline 12 & 1500 & 2000 & 1500 & 1000 & 155 & 135 & 35 & 5 & 0.01 & -5.64 & 27.2 & 27.9 & 35.9 & 37.7 & 27.2 & 27.9 & 35.8 & 37.7 \\
\hline 13 & 2000 & 850 & 1000 & 2000 & 140 & 120 & 70 & 30 & 0.00 & -9.15 & 23.7 & 25.5 & 35.4 & 45.0 & 23.7 & 25.5 & 35.4 & 45.0 \\
\hline 14 & 2000 & 1000 & 850 & 1500 & 145 & 135 & 75 & 45 & 0.00 & -12.93 & 19.4 & 20.3 & 37.3 & 53.7 & 19.4 & 20.3 & 37.3 & 53.6 \\
\hline 15 & 2000 & 1500 & 2000 & 1000 & 135 & 95 & 25 & 5 & 0.02 & -4.50 & 34.4 & 31.0 & 27.7 & 27.4 & 34.4 & 31.0 & 27.7 & 27.4 \\
\hline 16 & 2000 & 2000 & 1500 & 850 & 135 & 105 & 25 & 15 & 0.02 & -4.73 & 32.4 & 31.0 & 29.8 & 29.8 & 32.4 & 31.0 & 29.8 & 29.8 \\
\hline 17 & 850 & 850 & 2000 & 850 & 125 & 105 & 75 & 45 & 0.00 & -11.80 & 24.1 & 27.2 & 34.7 & 45.2 & 24.1 & 27.2 & 34.7 & 45.2 \\
\hline 18 & 850 & 1000 & 1500 & 1000 & 105 & 95 & 70 & 30 & 0.01 & -7.26 & 31.6 & 31.0 & 29.6 & 28.1 & 31.6 & 31.0 & 29.6 & 28.1 \\
\hline 19 & 850 & 1500 & 1000 & 1500 & 175 & 135 & 25 & 15 & 0.02 & -5.28 & 27.0 & 27.9 & 36.7 & 37.0 & 27.0 & 27.9 & 36.7 & 37.0 \\
\hline 20 & 850 & 2000 & 850 & 2000 & 150 & 120 & 25 & 5 & 0.02 & -4.96 & 29.5 & 29.7 & 33.6 & 33.8 & 29.5 & 29.7 & 33.6 & 33.8 \\
\hline 21 & 1000 & 850 & 2000 & 1000 & 165 & 135 & 40 & 30 & 0.01 & -6.34 & 25.0 & 26.2 & 37.9 & 39.8 & 25.0 & 26.2 & 37.9 & 39.8 \\
\hline 22 & 1000 & 1000 & 1500 & 850 & 160 & 120 & 65 & 45 & 0.00 & -10.42 & 20.4 & 24.2 & 38.6 & 47.5 & 20.4 & 24.2 & 38.6 & 47.5 \\
\hline 23 & 1000 & 1500 & 1000 & 2000 & 115 & 105 & 35 & 5 & 0.02 & -5.20 & 33.5 & 32.1 & 26.7 & 26.2 & 33.55 & 32.1 & 26.6 & 26.2 \\
\hline 24 & 1000 & 2000 & 850 & 1500 & 115 & 95 & 55 & 15 & 0.01 & -5.65 & 33.4 & 31.1 & 27.8 & 26.4 & 33.4 & 31.2 & 27.8 & 26.4 \\
\hline 25 & 1500 & 850 & 1500 & 1500 & 145 & 105 & 45 & 5 & 0.01 & -5.44 & 30.0 & 30.1 & 32.1 & 33.2 & 30.0 & 30.1 & 32.1 & 33.2 \\
\hline 26 & 1500 & 1000 & 2000 & 2000 & 125 & 95 & 45 & 15 & 0.01 & -5.41 & 33.6 & 31.0 & 28.2 & 27.6 & 33.6 & 31.0 & 28.2 & 27.6 \\
\hline 27 & 1500 & 1500 & 850 & 850 & 155 & 135 & 50 & 30 & 0.00 & -6.83 & 24.5 & 25.5 & 38.1 & 41.8 & 24.5 & 25.5 & 38.0 & 41.8 \\
\hline 28 & 1500 & 2000 & 1000 & 1000 & 130 & 120 & 55 & 45 & 0.00 & -9.08 & 25.4 & 26.4 & 37.9 & 40.1 & 25.4 & 26.4 & 37.8 & 40.1 \\
\hline 29 & 2000 & 850 & 1500 & 2000 & 145 & 135 & 35 & 15 & 0.01 & -5.53 & 27.7 & 27.9 & 35.7 & 36.8 & 27.7 & 27.9 & 35.7 & 36.8 \\
\hline 30 & 2000 & 1000 & 2000 & 1500 & 140 & 120 & 15 & 5 & 0.02 & -4.87 & 30.7 & 30.5 & 32.1 & 32.2 & 30.7 & 30.5 & 32.1 & 32.3 \\
\hline 31 & 2000 & 1500 & 850 & 1000 & 135 & 105 & 85 & 45 & 0.00 & -13.41 & 20.9 & 26.2 & 32.9 & 53.2 & 20.9 & 26.2 & 32.9 & 53.1 \\
\hline 32 & 2000 & 2000 & 1000 & 850 & 135 & 95 & 60 & 30 & 0.01 & -6.98 & 29.3 & 30.4 & 32.4 & 34.2 & 29.1 & 30.4 & 32.4 & 34.2 \\
\hline
\end{tabular}

According to the experience, the output node was set to 2 during the simulation of surge and sway. And the simulation of the anchor force was 8 . Both of the input nodes were set to 8 , and the number of hidden nodes, which led to the smallest global error, was set by cycle from 5 to 20. the $1 \sim 30$ groups were taken as training samples, 31 and 32 groups as a test sample to verify the generalization of BP neural network in Tab.6. Here only gave the movement of $0^{\circ}$ wave. After 940 training, the number of hidden nodes was 14; the latter after 2376 training, is 14. Both of he error is less than 0.01, which is target global error. The trained neural networks were tested with 31 and 32 groups in Tab.5. 
Tab.5 Relative Error of Simulation Training of $0^{\circ}$ wave

\begin{tabular}{|c|c|c|c|c|c|c|c|c|c|c|}
\hline \multirow{2}{*}{ num } & \multicolumn{10}{|c|}{ Relative error $(\%)$} \\
\cline { 2 - 24 } & Surge & Sway & P1 & P2 & P3 & P4 & S1 & S2 & S3 & S4 \\
\hline 31 & 2.43 & $\backslash$ & 5.0 & 11.43 & 5.4 & 11.18 & 13.7 & 7.68 & 12.6 & 12.12 \\
\hline 32 & 8.18 & $\backslash$ & 2.3 & 12.4 & 2.8 & 13.32 & 8.22 & 7.8 & 12 \\
\hline
\end{tabular}

From above results, it can be seen that the simulation can meet the engineering requirements. According to the specification of mooring system, the probability of occurrence of each wave angle in the actual condition was given in Tab.6, and the genetic algorithm is used to optimize the design. Take the anchor distance and the azimuth angle as the optimization variable, the displacement of surge and sway as the optimization target, the stress requirement of each anchor chain as the constraint condition.

Tab.6 Occurrence Probability of Different Wave

\begin{tabular}{|c|c|}
\hline Wave angle & The probability of occurrence $(\%)$ \\
\hline $0^{\circ}$ & 16.26 \\
\hline $45^{\circ}$ & 13.655 \\
\hline $90^{\circ}$ & 11.095 \\
\hline $135^{\circ}$ & 20.495 \\
\hline $180^{\circ}$ & 38.495 \\
\hline
\end{tabular}

Tab.7 Layout after Optimization

\begin{tabular}{|c|c|c|c|c|}
\hline Anchor chain & $\mathrm{P} 1$ & $\mathrm{P} 2$ & $\mathrm{P} 3$ & $\mathrm{P} 4$ \\
\hline Angle(after/before) & $-154 /-136$ & $-120 /-124$ & $-77 /-54$ & $-42 /-31$ \\
\hline $\begin{array}{c}\text { Distance between anchor and } \\
\text { guide hole(after/before) }\end{array}$ & $1506 / 1500$ & $850 / 1500$ & $850 / 1500$ & $2000 / 1500$ \\
\hline Anchor chain & $\mathrm{S} 1$ & $\mathrm{~S} 2$ & $\mathrm{~S} 3$ & $\mathrm{~S} 4$ \\
\hline $\begin{array}{c}\text { angle(after/before) } \\
\text { distance between anchor and } \\
\text { guide hole(after/before) }\end{array}$ & $154 / 151$ & $120 / 136$ & $77 / 62$ & $42 / 42$ \\
\hline
\end{tabular}

Due to the randomness of the initial population, the optimal solution based on the MATLAB genetic algorithm toolbox is different. So taking the optimal solution of 500 times calculations was shown in Tab.7. According to the optimized layout, the time domain calculation using Moses will optimize the results before. The comparison was shown in Tab.8.

Tab.8 Maximum Displacement Contrast between before and after Optimization

\begin{tabular}{|c|c|c|c|c|c|}
\hline \multirow{2}{*}{ Translational displacement } & \multicolumn{5}{|c|}{ Wave angle } \\
\cline { 2 - 6 } & $0^{0}$ & $45^{0}$ & $90^{0}$ & $135^{0}$ & $180^{0}$ \\
\hline before & 8.53 & 17.39 & 25.4 & 17.2 & 7.47 \\
\hline after & 9.09 & 16.36 & 19.04 & 14.15 & 6.83 \\
\hline
\end{tabular}

\section{Conclusion.}

We can get the following conclusions.

(1) Based on the results of Moses software, if the pre-tension of the anchor chain is improved, the displacement of the floating body will gradually be reduced.

(2) The error of the time domain movement and anchor force for the five waves angles based on BP neural network is not more than 15\% compared with Moses. It can meet the engineering needs. Therefore, it is considered feasible to use the neural network instead of Moses.

(3) After the anchor chain is optimized by genetic algorithm, the translational displacement of the other wave angles decreases with the increase of $0^{\circ}$ wave. In particular, the movement gets the most significant changes under $90^{\circ}$ wave. 
(4) The optimization method of mooring system based on BP neural network and genetic algorithm can provide a reference for the mooring arrangement of other floating structures at sea.

\section{Summary}

Based on the engineering practice, taking the azimuth and anchor position of the cable as the optimization variable, BP neural network and genetic algorithm are used to optimize the laying vessel working in the South China Sea. In this process, compared with the result of Moses software, BP neural network met the accuracy requirements on the basis of more time-saving. Whether it is the simulation of the sample, or its superior ability to predict are fully reflected. Limited to the established hardware conditions and the lack of knowledge of the author, the results still have some limitations to be further explored by scholars.

\section{Acknowledgement}

PROGRAM: Youth Fund of Wenhua College J02e0540211

\section{Reference}

[1] YU Long, TAN Jia Hua. Optimization of multi-component mooring line based on quasi-stationary method[J]. Ocean Engineering, 2005, 1 (in Chinese)

[2] MA Xiao Jian, SUN Zhao Cheng, ZHANG Zhi Ming, etal. Study on tension and movement of cable in mooring ship under wind and flow [J]. Waterway Port, 2010,7 (in Chinese)

[3] Mir Tareque Ali and Yoshiyuki Inoue. On hydrodynamic interaction between two rectangular barges floating side-by-side in regular waves. International Conference on Mechanical Engineering. 2005

[4] YAN Chuan Xu, QIAN Hong, XIANG Jun Yi, etal. Study on optimum design of pipe anchor positioning system[J]. Chinese Shipbuilding, 2010 (in Chinese)

[5] SU Xiao Yu, JIN Hong Zhang, HU Xiao Dong. Optimal parametric design of bulkhead vibration control for underwater structure[J]. Ocean Engineering, 2015(04)(in Chinese)

[6] XU Xiao Ying, YAN Hong Sheng, LIU Feng Di. Tension hybrid optimization algorithm for automatic mooring positioning system[J]. Ship Engineering, 2013(04) (in Chinese) 